\title{
Commissioning MATISSE: operation and performances
}

Petrov, Romain, Allouche, Fatmé, Matter, Alexis, Meilland, Anthony, Lagarde, Stéphane, et al.

Romain G. Petrov, Fatmé Allouche, Alexis Matter, Anthony Meilland, Stéphane Lagarde, Philippe Berio, Pierre Cruzalèbes, Florentin Millour, Sylvie RobbeDubois, Walter Jaffe, Karl-Heinz Hofmann, Jozsef Varga, Dieter Schertl, Leonard Burtscher, Klaus Meisenheimer, Alain Chelli, Gerard Zins, Julien Woillez, Markus Schöller, Bruno Lopez, "Commissioning MATISSE: operation and performances," Proc. SPIE 11446, Optical and Infrared Interferometry and Imaging VII, 114460L (30 December 2020); doi: 10.1117/12.2562569 


\title{
Commissioning MATISSE: operation and performances
}

\author{
Romain G. Petrov ${ }^{*}$, Fatmé Allouche ${ }^{\mathrm{a}}$, Alexis Matter ${ }^{\mathrm{a}}$, Anthony Meilland ${ }^{\mathrm{a}}$, Stéphane Lagarde ${ }^{\mathrm{a}}$, Philippe Bério ${ }^{\mathrm{a}}$, Pierre \\ Cruzalèbes $^{\mathrm{a}}$, Florentin Millour ${ }^{\mathrm{a}}$, Sylvie Robbe-Dubois ${ }^{\mathrm{a}}$, Walter Jaffe ${ }^{\mathrm{b}}$, Karl-Heinz Hofmann ${ }^{\mathrm{c}}$, Jozsef Varga ${ }^{\mathrm{b}}$, Dieter \\ Schertl $^{\mathrm{c}}$, Leonard Burtscher ${ }^{\mathrm{b}}$, Klaus Meisenheimer ${ }^{\mathrm{d}}$, Alain Chelli ${ }^{\mathrm{a}}$, Gérard Zins ${ }^{\mathrm{e}}$, Julien Woillez ${ }^{\mathrm{e}}$, Markus Schöller ${ }^{\mathrm{e}}$ and \\ Bruno Lopez ${ }^{\mathrm{a}}$. \\ a'Laboratoire Lagrange, Université Côte d'Azur, Observatoire de la Côte d'Azur, CNRS, Boulevard de l'Observatoire, CS \\ 34229, 06304 Nice Cedex 4, France. \\ beiden Observatory, Leiden University, Niels Bohrweg 2, NL-2333 CA Leiden, the Netherlands. \\ 'Max-Planck-Institut für Radioastronomie, Auf dem Hügel 69, D-53121 Bonn, Germany. \\ ${ }^{\mathrm{d}}$ Max Planck Institute für Astronomy, Königstuhl 17, D-69117 Heidelberg, Germany. \\ 'European Southern Observatory Headquarters, Karl-Schwarzschild-Straße 2, 85748 Garching bei München, Germany.
}

\begin{abstract}
MATISSE, the VLTI $2^{\text {nd }}$ generation spectro-interferometric L, M and N bands imager, has been commissioned from March 2018 to March 2020. It is open to the General User since April 2019. A complete analysis of its performances is given in this paper for MATISSE standalone (with UTs and ATs) and for the GRAVITY for MATISSE (GRA4MAT) mode (with ATs) where the GRAVITY fringe tracker is used to stabilize the fringes in MATISSE and hence improve its sensitivity and spectral coverage at high spectral resolution. This paper presents the key operation parameters of MATISSE and decomposes its performances in fundamental precision per spectral channel for all measurements and in broad band calibration errors on the accuracy of visibility and closure phase. It is intended to give the user a full description of the different errors that must be considered and weighted in the model fitting and image reconstruction. The first image reconstructions achieved by MATISSE are discussed. The performances demonstrated here in the full very broad spectral domain of MATISSE open a very large domain of scientific applications that includes but strongly expands quantitatively and qualitatively the initial science program of the first generation instrument MIDI and, combined with GRAVITY, offers an extremely powerful tool to characterize the temperature and composition of dusty and molecular components of YSOs, AGNs and evolved stars.
\end{abstract}

Keywords: Astronomy, Instrumentation, Optical long baseline interferometry, Thermal Infrared, Spectro-interferometry, Very Large Telescope Interferometer, VLTI, MATISSE.

\section{INTRODUCTION}

The Very Large Telescope Interferometer (VLTI) is currently the long baseline optical interferometer in operation with the largest collecting area and the highest sensitivity. It combines 4 fixed Unit Telescopes (UTs) with 8m diameter or 4 relocatable Auxiliary Telescopes with $1.8 \mathrm{~m}$ diameter. Its longest baseline is currently of $135 \mathrm{~m}$ and will soon be extended for ATs to the maximum value of $200 \mathrm{~m}$ allowed by the size of the platform at the top of mount Paranal [1]. The VLTI features two $2^{\text {nd }}$ generation instruments open the "General User", GRAVITY in the K band $[2,3]$ and MATISSE in the mid infrared covering the $\mathrm{L}, \mathrm{M}$ and $\mathrm{N}$ bands from 3 to $13 \mu \mathrm{m}$ [4,5]. GRAVITY contains a fringe tracker, the GFT that can be also used to stabilize the fringes recorded by MATISSE in the so called GRA4MAT (for GRAVITY for MATISSE) mode. MATISSE has been installed on the VLTI at the beginning of 2018 and has been included in the VLT(I) call for proposals for the first time in September 2018. Observations in open time started in April 2019. The first science results have just been published [6,7] and many others are in the final steps of redaction. The commissioning of MATISSE has started in March 2018 and was scheduled to be concluded in May 2020. In spite of the delay in the last two commissioning runs scheduled in spring 2020 and postponed by the Paranal shutdown due to the covid-19 crisis, the analysis of MATISSE performances is nearly complete. This paper presents the MATISSE performance analysis method and the key results that the General-User should have in mind when (s)he wants to analyze the feasibility and the potential of an observing

\footnotetext{
* Romain.PETROV@univ-cotedazur.fr
}

Optical and Infrared Interferometry and Imaging VII, edited by Peter G. Tuthill, Antoine Mérand, Stephanie Sallum, Proc. of SPIE Vol. 11446, 114460L · (c) 2020 SPIE

CCC code: $0277-786 \mathrm{X} / 20 / \$ 21 \cdot$ doi: $10.1117 / 12.2562569$ 
proposal. The limiting performances given here are very close to these offered in the ESO Call for Proposals (CfP) and this paper is aimed at explaining how they have been obtained and how they can be interpreted and developed.

The main science goals of MATISSE are described in [5] and [8]. They are mainly based on angular measurements, and ideally images, of dust and molecules with temperatures ranging from 200 to $1500 \mathrm{~K}$. The very broad spectral coverage of MATISSE offers unique constraints on the temperature distribution and mineralogy of the dust in YSOs and AGNs. In the $\mathrm{L}$ band it has also access to a large collection of ionized hydrogen lines that can be used for the gas-dust interaction in YSOs, AGNs and active stars with circumstellar environments.

This paper focusses on the performances and limitations of MATISSE standalone as well as in combination with the GFT. However, it is worth having in mind that as a VLTI instrument, MATISSE is also limited by the Adaptive Optics of the VLTI, which sets a V limiting magnitude (NAOMI on ATs with $\mathrm{V}_{\lim } \sim 12$ and MACAO on UTs with $\mathrm{V}_{\text {lim }} \sim 14$ ) and by the necessity to track the images in the focal laboratory in spite of the drifts introduced by the long airpath in the delay line tunnels. This set a $\mathrm{K}$ band limiting magnitude even when the GFT is not used.

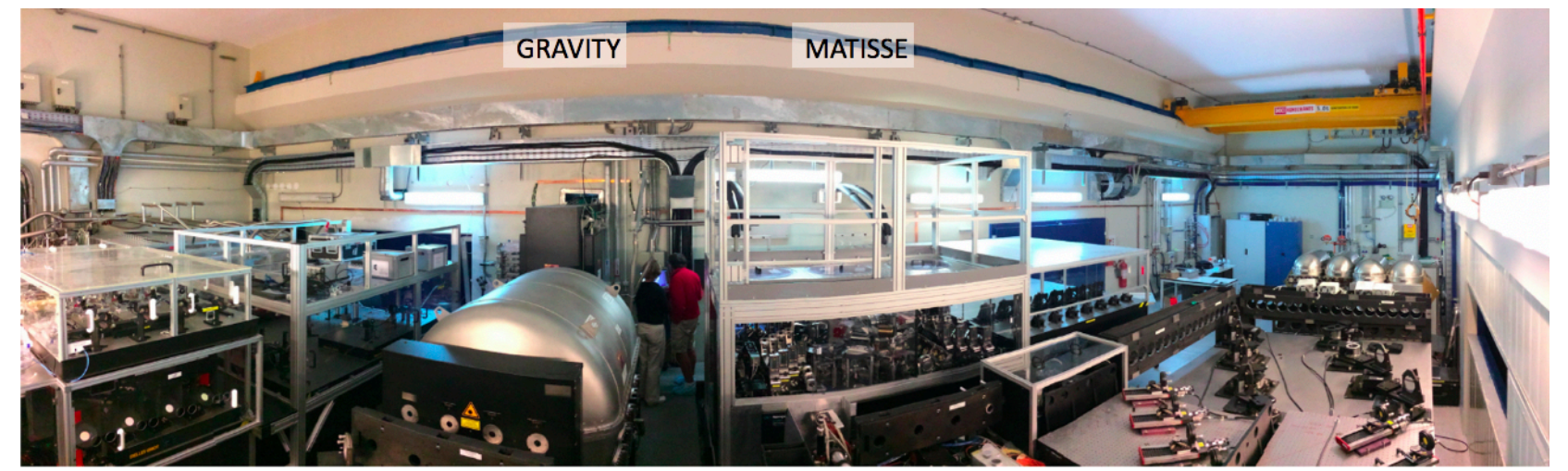

Figure 1. MATISSE (center right) and GRAVITY (center left) in the VLTI focal laboratory.

In section 2 we describe MATISSE and its measurements in order to introduce the way their errors depend from the key instrument parameters. Section 3 gives the method to compute and validate the effect of fundamental noise on the precision of all measurements. It also describes the calibration errors that affect the accuracy of the visibility and closure phase. Section 4 describes the combination of all these factors in a global performance estimate and shows how this global performance estimate have been validated before giving a synthesis of the performances of MATISSE. Finally, section 5 describes the performance improvement permitted by the GFT on ATs.

\section{MATISSE}

\subsection{Instrument concept and key features}

MATISSE is mid infrared imaging spectro-interferometer covering the $\mathrm{L}, \mathrm{M}$ and $\mathrm{N}$ spectral bands, respectively from 2.9 to $4.1 \mu \mathrm{m}, 4.5$ to $5 \mu \mathrm{m}$ and 8 to $13 \mu \mathrm{m}$. These spectral bands and the maximum current baseline of the VLTI corresponds to resolutions ranging from 4.5 to 15 milliarcseconds (mas). It is based on an all-in-one concept, similar to this of the firstgeneration instrument AMBER [9]. All beams are focused inside a common Airy disk that contains 6 fringe patterns, one for each baseline. The fringe patterns are separated in Fourier space by the a non-redundant spacing on the internal pupils in MATISSE. The fringed Airy disk is dispersed by a spectrograph in the direction perpendicular to the internal baselines yielding a dispersed fringe interferogram in a $x-\lambda$ frame. This interferogram can be completed by individual dispersed photometric beams, each containing the flux and the spectrum of the source seen by each individual aperture.

MATISSE is divided in 3 main components that are the Warm Optics table and the L-M and $\mathrm{N}$ band cryostats.

The Warm Optics components shape the beams that must be injected in the cryostats to ensure the proper sampling of the fringes and photometric beams. They co-align MATISSE with the VLTI. They split the light between the L-M and the N bands. They include a reference source that is used for the automatic alignment of all MATISSE components, for the cophasing of all MATISSE beams and for the calibration of the detectors and the instrumental complex visibility. They also contain two devices intended to improve the precision of MATISSE measurements. 
The Beam Commutation Device (BCD) [10] switches beams 1-2 and 3-4 in 2-1 and 4-3 without loss of alignment or cophasing and in less than 4 seconds. This operation allows to invert all MATISSE closure phases and the differential phases on baselines 1-2 and 3-4 without changing the instrumental term that they contain. Then the subtraction of measures with different BCD setups allows to eliminate the instrument errors on the closure and relevant differential phases that vary slower than the $\mathrm{BCD}$ calibration cycle, which is typically based on one commutation per mn. This removes the closure phase and differential phase errors resulting from the small evolution of the detector and the residual cross talks between beams and baselines.

The fast OPD modulation of each beam during the piston coherence time of the atmosphere is used to eliminate the contamination of the fringe peaks by the rapid variations of the background in the $\mathrm{N}$ band. The modulation cycle is executed in $240 \mathrm{~ms}$ over $10 \mathrm{~N}$ band frames of $20 \mathrm{~ms}$. The modulation amplitudes on each beam allow to demodulate each baseline individually while leaving all other baselines and the photometric low frequency component modulated by a zero-mean signal.

Each one of the Cold Optics cryostats contains a spatial filtering using pinholes and pupil masks that allow MATISSE to be very close to a single mode beam combiner. It contains also the optional separation between interferometric and photometric beams and the dispersive elements that define the instrument spectral resolutions. They allow also a nondispersed mode that can be used for image acquisition and the possibility for form pupil images on the detector used to the internal alignment of the instruments.

The L-M band and the $\mathrm{N}$ band spectrograph allow the following spectral resolutions.

Table 1. Spectral modes and resolutions of MATISSE

\begin{tabular}{|llll|}
\hline Spectral Mode & Wavelength range & Resolution & Pixels per $\lambda / \mathrm{D}$ \\
LR-LM & $2.9-5 \mu \mathrm{m}$ & 31.5 & \\
MR-LM & $2.9-5 \mu \mathrm{m}$ & 499 & 5 (at $3.5 \mu \mathrm{m})$ \\
HR-L & $2.9-4.1 \mu \mathrm{m}$ & 979 & \\
\hline VHR-LM & $3.9-5 \mu \mathrm{m}$ & 3370 & 7 (at $4.7 \mu \mathrm{m})$ \\
\hline LR-N & $8-13 \mu \mathrm{m}$ & 31.5 & 7 (at $10.5 \mu \mathrm{m})$ \\
HR-N & $8-13 \mu \mathrm{m}$ & 218 & \\
\hline
\end{tabular}

The LM spectrograph uses and Hawaii-2 detector with $2048 \times 2048$ pixels that can be used in a "slow-read" mode with a detector read out noise of about $15 \mathrm{e}^{-}$per pixel and per read and a fast "read-mode" with about $75 \mathrm{e}^{-}$per pixel and per read. The "fast-read" mode is used only in low spectral resolution (LR-LM) for targets brighter than $\sim 300 \mathrm{Jy}$ in L to avoid detector saturation. In "slow-read" mode the spectral window that can be read in an atmospheric coherence time (less than $120 \mathrm{~ms}$ in L) is of $0.2 \mu \mathrm{m}$ in MR-LM and $0.1 \mu \mathrm{m}$ in HR-L. Reading the full Hawaii detector needs nearly $1 \mathrm{~s}$ and this can be used only when an external fringe tracker stabilizes the fringes observed by MATISSE.

The $\mathrm{N}$ band spectrograph contains an Aquarius 1024x1024 detector. In low spectral resolution (LR-N) the frame time is set to $20 \mathrm{~ms}$ to avoid saturation by the thermal background. In "high" (HR-N) spectral resolution a frame time of $75 \mathrm{~ms}$ allows to read the full spectral 8-13 $\mu \mathrm{m}$ window.

The internal alignment and cophasing of MATISSE is realized automatically from observations of its internal reference source. This implies the use of a large number of actuators both on the warm and cold optics bench. A careful reliability analysis made during the design of the instrument showed that this large number of actuators will not be a cause of instability or breakdowns. This estimation has been fully confirmed by the first two years of operations of MATISSE. This automated alignment procedure is an enormous progress for the operation and maintainability of MATISSE, that is considered as "easy to operate" by the Paranal teams.

\subsection{MATISSE measurements}

Like any spectro-interferometric instrument since AMBER [9], MATISSE yields the following measurements:

- The complex coherent flux as a function of wavelength $C_{i j}(\lambda)$ for each baseline $B_{i j}$.

$$
C_{i j}(\lambda)=\left\langle\hat{I}\left(B_{i j} / \lambda, \lambda\right)\right\rangle=\sqrt{n_{* i}(\lambda) n_{* j}(\lambda)} V_{i j}(\lambda) e^{i \varphi_{i j}(\lambda)}
$$


where $\hat{I}\left(B_{i j} / \lambda, \lambda\right)$ is the Fourier transform of the interferogram $I(x, \lambda)$ at the spatial frequency $B_{i j} / \lambda, n_{* i}(\lambda)$ is the source flux contribution of beam $i$ to the interferogram and $V_{i j}(\lambda)$ and $\varphi_{i j}(\lambda)$ are the modulus and phase of the complex visibility on the baseline $i j$ at the wavelength $\lambda$. As $\varphi_{i j}(\lambda)$ randomly changes because of the atmospheric piston, the complex coherent flux can be integrated beyond the atmospheric coherence time only if the phase delay is measured and corrected in each frame either from MATISSE data itself (in the data reduction) of from an external fringe tracker (in real time). When this is impossible, we can use the phase independent squared modulus of the complex coherent flux

$$
\left|C_{i j}(\lambda)\right|^{2}=<\left|F T\left[I_{i j}(\lambda)\right]\right|^{2}>=n_{* i}(\lambda) n_{* j}(\lambda) V_{i j}(\lambda)
$$

- The source spectrum $S(\lambda)$ can be obtained from the photometric measurements:

$$
S(\lambda)=\frac{\sum_{i}<n_{* i}(\lambda)>}{\sum_{i}<n_{* i}\left(\lambda_{C}\right)>}
$$

where $\lambda_{C}$ represents a spectral channel in the continuum.

- The source visibility $V_{* i j}(\lambda)$ can be obtained from a combination of the coherent flux and photometric measurements

$$
V_{* i j}(\lambda)=\frac{\left|c_{i j}(\lambda)\right|}{V_{I i j}(\lambda) \sqrt{\left\langle n_{* i}(\lambda) n_{* j}(\lambda)>\right.}}
$$

where $V_{I i j}(\lambda)$ is the instrumental visibility. As $V_{I i j}(\lambda)$ can change quite rapidly with seeing and also instrument features such as vibrations in the VLTI, it is necessary to use a calibration source with known $V_{* i j}(\lambda)$ to measure $V_{I i j}(\lambda)$ and hence obtain an accurate measure of $V_{* i j}(\lambda)$ on the science source.

MATISSE does not have access to an absolute phase reference (but for the very specific and rare case of a calibrator close enough to the science to appear in the MATISSE $4 \lambda / \mathrm{D}$ field) and it must use the source closure phase and the color differential phases.

- The closure phase is deduced from the coherent flux bispectrum

$$
\psi_{i j k}(\lambda)=\arg \left[C_{i j}(\lambda) C_{j k}(\lambda) C_{k i}(\lambda)\right]
$$

In theory that closure phase is independent of instrumental features in a single mode experiment. In practice there is an instrumental closure phase due to cross-talks between beams and baselines [11]

$$
\psi_{i j k}(\lambda)=\psi_{* i j k}(\lambda)+\psi_{I i j k}(\lambda)
$$

The instrumental term $\psi_{I i j k}(\lambda)$ can be calibrated with a calibrator or using the BCD that allows to exchange $i$ and $j$ at the entrance of MATISSE. We then have pairs of measurements with different BCD setups:

$$
\begin{gathered}
\psi_{\text {OUTijk }}(\lambda)=\psi_{* i j k}(\lambda)+\psi_{\text {Iijk }}(\lambda) \\
\psi_{\text {INijk }}(\lambda)=\psi_{* j i k}(\lambda)+\psi_{I i j k}(\lambda)=-\psi_{* i j k}(\lambda)+\psi_{I i j k}(\lambda) \\
\psi_{* i j k}(\lambda)=\frac{\psi_{\text {OUTijk }}(\lambda)-\psi_{I N i j k}(\lambda)}{2}
\end{gathered}
$$

The terms "IN" and "OUT" are a conventional way to name the two BCD setups, but the beams see the same number of mirrors in each one of these setups.

- The differential phase is the difference between the phase in a given spectral channel and the phase in a reference channel usually obtained from an average of the coherent flux over wavelength.

$$
\phi_{D_{i j}}(\lambda)=\arg \left[C_{i j}(\lambda)\left\langle C_{i j}^{*}(\lambda)\right\rangle_{\lambda}\right]-2 \pi P_{i j}(\lambda) / \lambda
$$

where $P_{i j}(\lambda)$ is the residual chromatic group delay estimated from a fit of $\arg \left[C_{i j}(\lambda)\left\langle C_{i j}^{*}(\lambda)\right\rangle_{\lambda}\right]$, ideally only on channels where the source is known or unresolved, like for example the continuum on each side of an emission line. That group delay can be developed in an achromatic term $P_{P i j}$ and a chromatic term $P_{C i j}(\lambda)$.

$$
P_{i j}(\lambda)=P_{P i j}+P_{C i j}(\lambda)
$$

The achromatic term $P_{P i j}$ results from the imperfect correction of the atmospheric piston in each frame. The chromatic term results from the chromatic variations of the air index and from the differences in air path seen by each beam. Currently we fit and correct only the achromatic term in each instrument band, from 3.1 to $3.9 \mu \mathrm{m}$ for the L\&M observations and 
from 8 to $10 \mu \mathrm{m}$ for the $\mathrm{N}$ observations. This gives the differential precision that is presented in this paper on the measured differential phase:

$$
\phi_{M D_{i j}}(\lambda)=\arg \left[C_{i j}(\lambda)\left\langle C_{i j}^{*}(\lambda)\right\rangle_{\lambda}\right]-2 \pi P_{P i j} / \lambda
$$

The modeling and calibration of the chromatic term $P_{C i j}(\lambda)$ is a work in progress that is expected to increase very substantially the differential phase accuracy.

- The differential visibility is a self-referenced visibility measurement given by

$$
\phi_{D_{i j}}(\lambda)=\frac{\Re\left[c_{i j}(\lambda)\left\langle c_{i j}^{*}(\lambda)\right\rangle_{\lambda}\right]}{\left\langle\left|c_{i j}(\lambda)\right|^{2}\right\rangle_{\lambda}}
$$

\subsection{Observing modes}

The modes of MATISSE are set by the method used to calibrate the photometry. In the SiPhot (Simultaneous Photometry) mode, a third of each beam is deflected to a photometric beam that appears on the same detector frame than the interferometric beam. In L, like in $\mathrm{K}$ with AMBER, there are fast photometric variations introduced by the fluctuation of the Strehl ratio of the partially correcting adaptive optics with strongly seeing dependent amplitude. On the other hand, the background variations are fainter and slower, and it should be possible to estimate them from a record of the sky background before and after the interferometric observation. The HighSens (High Sensitivity) has been inspired by MIDI. In this mode all the flux is concentrated in the interferometric channel and the photometry is analyzed sequentially by closing all beams but one. The HighSens mode main justification is that MIDI showed that a lot of spatial useful information can be obtained from the coherent flux alone which give access to the many sources that are too faint for good photometric and hence absolute visibility measurements while they are bright enough for coherent flux estimates. The difference comes from the fact that the strong and very variable background in $\mathrm{N}$ has no spatial coherence. The coherent flux SNR depends from the background photon noise, but it is almost unsensitive to the changes in background intensity that dominated the photometric measurements. In HighSens the source photometry is estimated sequentially, and the background is measured and corrected using telescope chopping. That source photometry is sensitive to changes in AO performances but they are quite limited in the $\mathrm{N}$ band.

MATISSE can use both SiPhot and HighSens in the two cryostats. However, the tests of MATISSE in laboratory showed that the SiPhot mode is hardly applicable to the Aquarius detector that has a linearity and a systematic noise which can be correctly calibrated only in a small flux range and on the same pixels.

We therefore decided to use MATISSE in the so-called Hybrid mode:

- The L-M cryostat is set in SiPhot mode and records interferometric and photometric channels simultaneously.

- The $\mathrm{N}$ cryostat is in HighSens mode and records only the interferometric channel.

- The so-called interferometric observation is executed without chopping. It yields interferometric and photometric observations without chopping in $\mathrm{L}$ and $\mathrm{M}$ and interferometric observations without chopping in $\mathrm{N}$.

- The standard MATISSE Observing Block (OB) contains 4 exposures of $1 \mathrm{mn}$, each in one of the $4 \mathrm{BCD}$ configurations that correspond to the following dispositions of the input beams: 1-2-3-4 (out-out), 2-13-4 (in-out), 1-2-4-3 (out-in) and 2-1-4-3 (in-in).

- This set of 4 exposures constitutes a "BCD cycle". It can be repeated several times, at users will, to increase the precision of all measurements and the accuracy on closure phase (as explained below)

$\circ$ The limit of $1 \mathrm{mn}$ per exposure is set by many parameters, the main one being the manageability of data transfers.

- During the so-called photometric observation, we record 8 exposures with all $\mathrm{N}$ band shutters but one closed with chopping.

$\bigcirc$ The photometry of each beam is measured in $\mathrm{N}$ with chopping and on the interferometric channel pixels with the corresponding BCD in position "in" and in position "out"

$\circ$ During these exposures, the L-M detector records the interferometric and photometric channels with chopping.

\subsection{Standard Observing Block}

The standard MATISSE OB contains:

- the telescopes preset, which includes pointing, finding a guide star and locking the active optics for the UTs, and finally closing the AO loops; 
- the acquisition of the images in the VLTI focal laboratory and the closing of the loop for image tracking in the laboratory (called "Coude Laboratory Guiding");

- the preset of the Delay Lines;

- the acquisition of the fringes by MATISSE or by the GRAVITY Fringe Tracker;

- 2 exposures of $30 \mathrm{~s}$ on the sky (one with BCD "out-out" and one with BCD "in-in")

- 4 exposures of $1 \mathrm{mn}$ on target without chopping (the $\mathrm{N}$ band interferometric exposures)

$\circ \quad$ that are also L-M band interferometric and photometric exposures without chopping

- 8 exposures of $1 \mathrm{mn}$ on target with chopping (the $\mathrm{N}$ band photometric exposures)

$\circ \quad$ that are also L-M band interferometric and photometric exposures with chopping

Given the small overheads between exposure, the minimum duration of this standard OB is $\sim 22 \mathrm{mn}$. ESO proposes an average duration of $25 \mathrm{mn}$.

An observing block can contain more than $1 \mathrm{BCD}$ cycle. Its length will then be increased by $\sim 5 \mathrm{mn}$ per BCD cycle. It can also be executed without "photometric" observations, when the sources are anyway too faint for visibility measurements or in higher spectral resolution modes where only wavelength differential measures are needed. An observing block without "photometry" is $\sim 10 \mathrm{mn}$ shorter.

\section{PERFORMANCE ANALYSIS}

\subsection{Performance criteria}

The limits of MATISSE are set by many VLTI parameters such as the limiting magnitude in V to close the loop of the adaptive optics (V 12 with NAOMI on ATs and V 14 with MACAO on UTs) and the K band limiting magnitude for image tracking in the VLTI focal laboratory. The parameters specific to MATISSE are its capability to acquire images and fringes either in $\mathrm{L}$ or in $\mathrm{N}$ and to maintain the fringes near the center of the coherence length with its internal group delay tracker. Finally, MATISSE observations are useful only if the data can be processed and provide good quality measurements. We checked the limiting flux for all these operations and found that the criterium on the quality of the processed data is by far the most demanding. We agreed with ESO that the MATISSE sensitivity limit are the coherent fluxes in Jy that allow the following precision or accuracy on each measure per spectral channel and per mn of observation.

- Visibility accuracy: $\sigma_{V}=0.1$

- Closure phase accuracy: $\sigma_{\psi}=5^{\circ}$

- Differential phase precision: $\sigma_{\varphi}=4^{\circ}$

- Coherent flux SNR: $C / \sigma_{C}=10$

These criteria are quite demanding. Better performances can be obtained by binning spectral channels to obtain broad band observations or by combining several observations. However, MATISSE users should keep in mind that the precision of

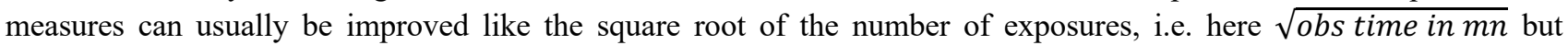
calibration errors can be improved only with the square root of the number of calibration cycles i.e. typically $\sqrt{\text { obs time in } h}$ for the most demanding absolute visibility calibration. For closure phase, the calibration cycle is the BCD cycle that takes typically $5 \mathrm{mn}$ and the closure phase accuracy increases like $\sqrt{\text { number of BCD cycles. }}$.

The visibility and closure phase accuracy given above is sufficient for image reconstruction on resolved targets. The quality of these images will generally be much more sensitive to the u-v coverage than to improvements of the closure phase and visibility accuracy beyond these limits. The coherent flux and differential measures allow only model dependent angular constraints on the source, at least with the current state-of-the-art image reconstruction algorithms.

In the next sections we will analyze the fundamental noise error that affect the precision of all measurements, the broad band seeing dependent and flux independent calibration errors that affect the accuracy of the visibility and closure phase and the flux dependent broad band photometric calibration error that affects only the visibility accuracy.

\subsection{Fundamental noise computation}

All MATISSE measurements are derived from the complex coherent flux estimates $C_{i j}(\lambda)$. The error on the coherent flux is set by the fundamental noises that are the source and the background photon noise and the detector noises that in MATISSE are dominated by the Read-Out Noise per pixel and per read. The variance of the coherent flux per spectral channel and per frame is given by [18]:

$$
\sigma_{C_{i j}}^{2}=N_{p} \sigma_{R}^{2}+\sum_{i=1}^{N_{T}}\left(n_{* i}+n_{B i}\right)
$$


with $n_{* i}$ and $n_{B i}$ for the numbers of source and background photons in beam $i, N_{T}$ is the number of telescopes, $N_{p}$ the number of pixels sampling the signal and $\sigma_{R}^{2}$ the variance of the read-out-noise. The average coherent flux SNR per channel and per frame is:

$$
S N R_{C_{i j 1}}=\frac{C_{i j 1}}{\sigma_{C_{i j 1}}}=\frac{\sqrt{n_{i *} n_{j *}} V_{i j}}{\sqrt{N_{p} \sigma_{R}^{2}+\sum_{i=1}^{N_{T}}\left(n_{* i}+n_{B i}\right)}}
$$

When $N_{\lambda}$ spectral channels and $N_{F}$ frames are added coherently, the coherent flux SNR becomes

$$
S N R_{C_{i j}}=S N R_{C_{i j 1}} \sqrt{N_{\lambda} N_{F}}
$$

An incoherent integration of channels or frames, i.e. an addition of interferogram power spectrum would yield

$$
S N R_{C_{i j}^{2}}=\frac{\left({ }^{S N R} C_{i j}\right)^{2}}{\sqrt{N_{\lambda} N_{F}\left[1+2\left(S N R_{C i j}\right)^{2}\right]}}
$$

which becomes extremely inefficient when $S N R_{C_{i j}}<1$. That sets a practical limit to incoherent integration modes. Above that limit, the incoherent addition of quadratic quantities has the advantage to be nearly unsensitive to tracking errors. The phase of the coherent flux has a precision

$$
\sigma_{\varphi_{i j}} \simeq \frac{1}{\sqrt{2} S N R C_{i j}}
$$

This approximation is correct up to $\sigma_{\varphi_{i j}} \simeq 1 \mathrm{rad}$. It is a good approximation of the fundamental error on the differential phase, as the errors introduced by the much broader reference channel can generally be neglected.

When the coherent flux phase precision per frame $\sigma_{\varphi_{i j 1}}<1 \mathrm{rad}$, the closure phase precision is given by

$$
\sigma_{\psi_{i j k}}^{2} \simeq \sigma_{\varphi_{i j}}^{2}+\sigma_{\varphi_{j k}}^{2}+\sigma_{\varphi_{k i}}^{2} \simeq 3 \sigma_{\varphi}^{2}
$$

When $\sigma_{\varphi_{i j 1}}>1 \mathrm{rad}$ the closure phase error has a more complex expression based on the analysis of the bispectrum SNR. This analysis is beyond the scope of this paper. In practice, we have found that a two terms development fits our measures with an accuracy sufficient to predict the closure phase error.

$$
\sigma_{\psi}=\sigma_{\varphi} \sqrt{3}+\alpha \sigma_{\varphi}^{3}
$$

Where $\alpha$ is constant for a given MATISSE set-up that set from the measured dispersion of the closure phase estimators. The closure phase precision very rapidly decreases below the $\sigma_{\varphi} \sim 1 \mathrm{rad}$ limit.

It is therefore critical to use the maximum number of channels and frames that can be coherently integrated. In the following we will give the results for coherent binning of the spectral columns in one spectral channel and for coherent integration of frames over the atmospheric coherence time.

The absolute visibility is deduced from the coherent flux through a photometric correction $V_{i j}(\lambda)=C_{i j} / \sqrt{n_{i *} n_{j *}}$ and its fundamental noise also depends from the precision of the estimates of $n_{i *}$ and $n_{j *}$ :

\subsection{Fundamental noise measurement}

$$
\sigma_{V_{i j}^{2}}=V_{i j}^{2}\left(\frac{\sigma_{C_{i j}^{2}}^{2}}{C_{i j}^{2}}+\frac{\sigma_{n_{* i}}^{2}}{n_{* i}{ }^{2}}+\frac{\sigma_{n_{* j}}^{2}}{n_{* j}{ }^{2}}\right)^{1 / 2}
$$

To use the expressions of the previous section to evaluate the fundamental noise errors we have updated the instrument parameters like the background and source fluxes as they depend from the transmission and the emissivity of optics, the read-out-noise and the instrumental contrast measured in laboratory or on sky. To validate these estimates, we have observed in each mode a large number of calibrators with different magnitudes, selected from the MDFC catalogue [12] to be very likely centrosymmetric sources (hence with zero differential and closure phase) that can be modelled by a disc. The diameter of the calibrators is estimated from their color indexes and a correction is applied to the measured visibility. To remove the effect of the broad band errors, which are separately analyzed in the next two sections, we fit the measurement $m(\lambda)$ with a low order polynomial function $f(\lambda)$ adapted to the measurement behavior (usually a $2^{\text {nd }}$ order function). Then we compute the measured standard deviation $\sigma_{M}(\lambda)$ of the residual $m(\lambda)-f(\lambda)$ and we plot the predicted and measured values on the same graph as illustrated in figures 2 . We fine tune the instrumental parameters (mainly the actual transmission) to have a computed value going through the median of measured points. This adjusted transmission is in agreement with the transmission measured in laboratory combined with the estimated VLTI transmission within $50 \%$. This is a remarkable agreement if we consider the accuracy of laboratory transmission measurements especially for the very large set of VLTI optics that evolve between coatings. Then the adjusted computed fundamental noise error is used to predict the precision of the measurement on any source given its coherent flux. This analysis has been made for all modes of MATISSE, for ATs and UTs. 

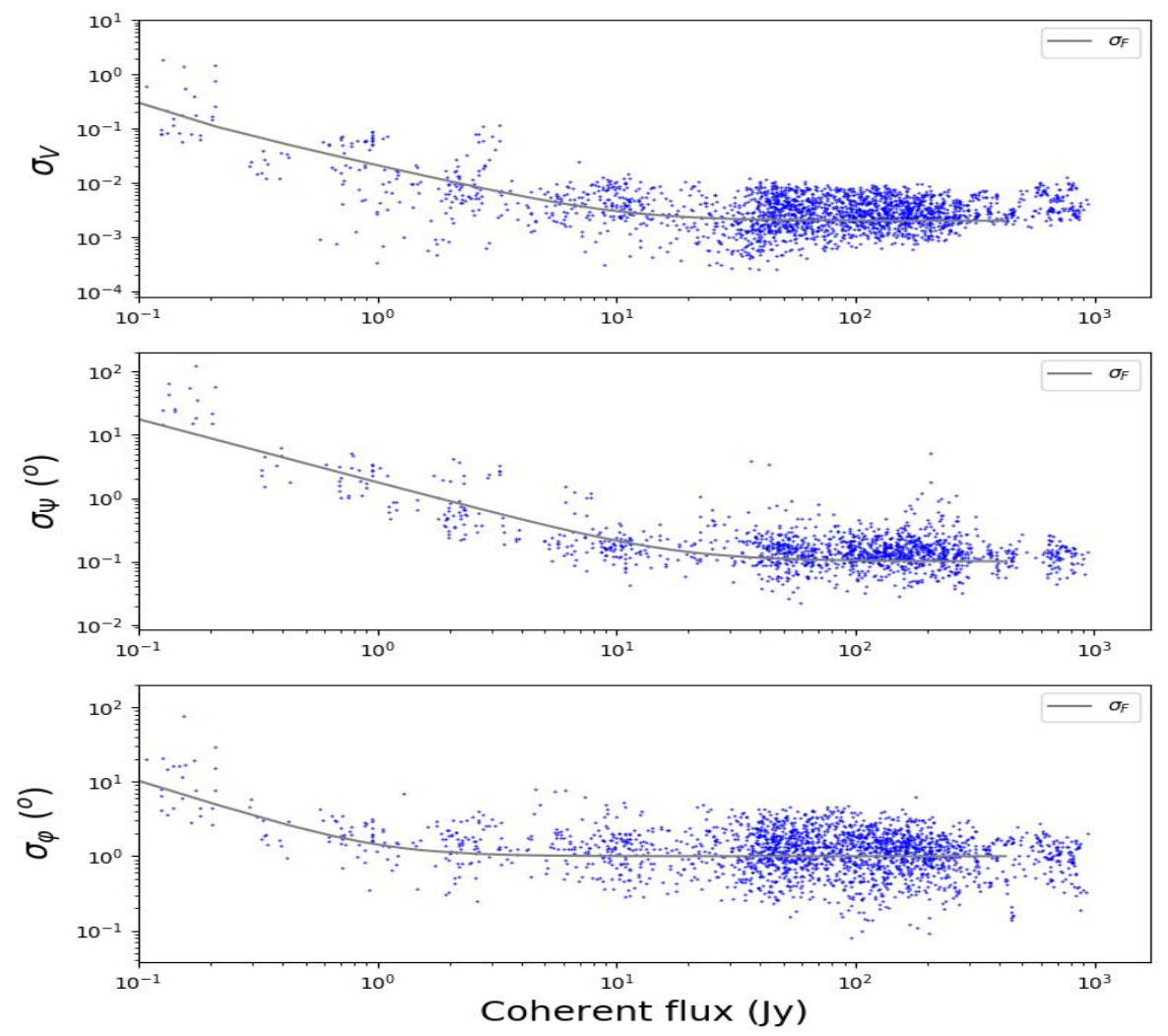

Figure 2. Illustration of the fundamental noise measurement and prediction in the case of $\mathrm{L}$ band observations at low spectral resolution with the ATs. We plot the measured fundamental noise per spectral channel (blue dots) as a function of the source coherent flux for the visibility (top), the closure phase (middle) and the differential phase (bottom). The grey line shows the value of the noise prediction given by the formulas in section 3.2. In these plots we merge all baselines, AT configurations and seeing conditions. About $80 \%$ of the measured values are within a factor 2 of the computed value.

On UTs we had much less commissioning time and we use the smaller number of available measures to set the conversion factors between ATs and UTs. In L the conversion factor is of the order of 14, which is below the expected surface ratio of 20. This indicates that the NAOMI adaptive optics deliver a higher and/or more stable Strehl ratio than the MACAO adaptive optics on UTs or that the UT Coude train needs to be recoated. In N the conversion factor is of the order of 30 , which indicates that the background noise is higher on ATs than on UTs in spite of the fact that on a single mode instrument the solid angle on the sky is independent of the telescope diameter.

The agreement between the computed and measured precision is good at low flux but the measured precision reaches a plateau at high flux and stops to decrease when the source brightness increases. To account for that we add a flux independent variance to the computed variance, that sets a lower limit to the precision of measurements per minute of observation that is given in table 2 below.

Table 2. Best (i.e. minimum) values of MATISSE errors at high flux. For the differential phase, these values are given for a broad reference channel. For narrow continuum-line-continuum measurement in L, the differential phase can be as precise as $0.2^{\circ}$.

\begin{tabular}{|cccc|}
\hline Measure & $\mathbf{L}$ & $\mathbf{M}$ & $\mathbf{N}$ \\
Visibility & $310^{-3}$ & $510^{-3}$ & $310^{-3}$ \\
Closure Phase & $0.1^{\circ}$ & $0.2^{\circ}$ & $0.1^{\circ}$ \\
Differential Phase & $1^{\circ}$ & $1^{\circ}$ & $0.2^{\circ}$ \\
\hline
\end{tabular}


The limits on the Visibility and Closure phase set a fundamental limit on the dynamical range of reconstructed images of the order of 300. This is quite lower than the limit set by the $\mathrm{u}-\mathrm{v}$ coverage that can be achieved in practice by a 4 apertures interferometer. The differential phase precision plateau sets a more severe limitation on the detection of faint companions, of the order of 1/300 in L and $\mathrm{M}$ and 1/1500 in N. This limit is due to the imperfect calibration of the chromatic OPD, that is the next frontier in MATISSE calibration and data processing.

\subsection{Instrumental visibility changes with seeing}

The changes in seeing conditions and some instrumental features, like transient telescope vibrations, affect the instrument + atmosphere instrumental response. This is illustrated in figure 3 showing the variation of the instrument visibility with seeing and atmospheric coherence time $\tau_{0}$.
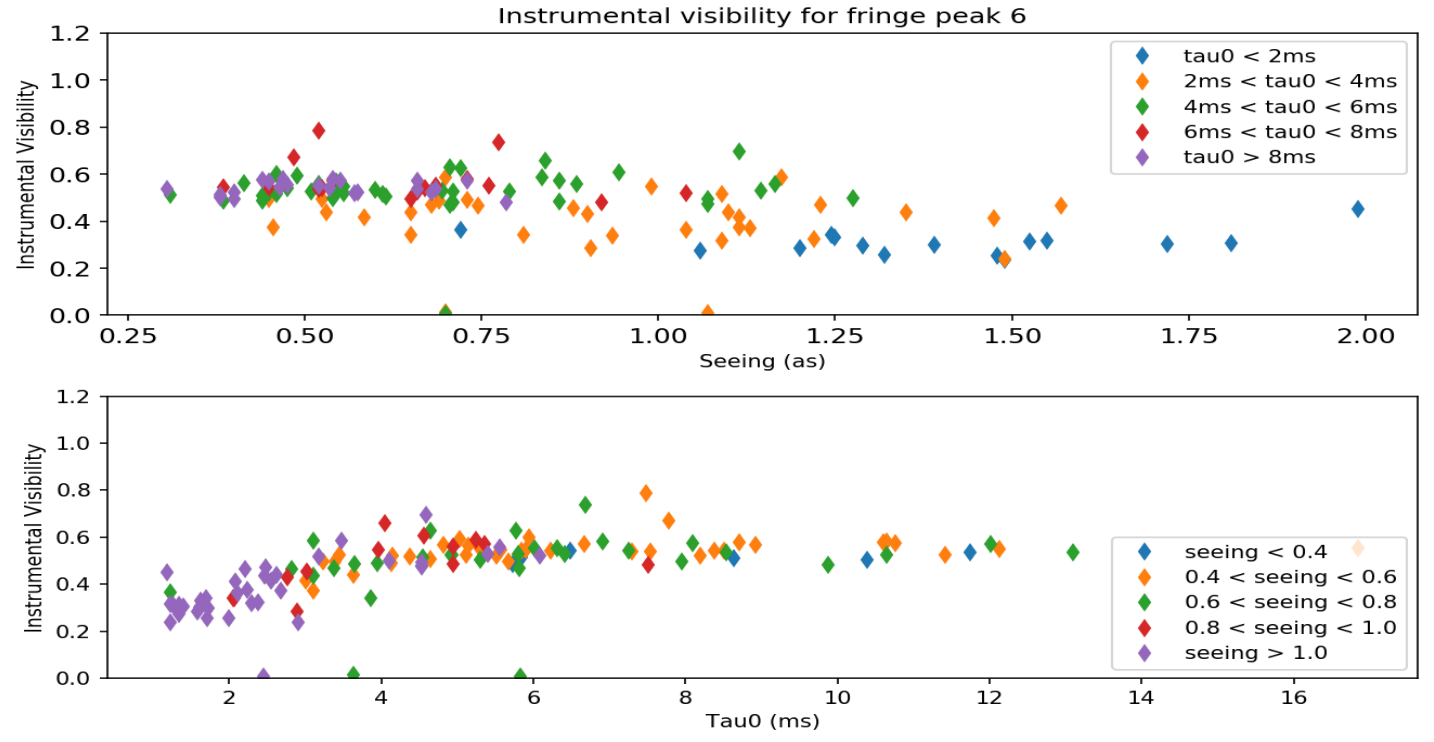

Figure 3. Variation of the instrument + atmosphere visibility as a function of seeing in arcseconds (top) and atmospheric coherence time in the visible in $\mathrm{ms}$ (bottom). In the top figure the colors also indicate the coherence time range, while in the bottom figure they indicate the seeing range. The observations were made in the $\mathrm{L}$ band, from 3.1 to $3.9 \mu \mathrm{m}$ with a frame time DIT=75 ms. As a wide range of magnitudes has been used, some measurements are sensitive also to fundamental noise, and to errors in calibrator diameter estimates and corrections.

The instrumental visibility has been estimated on calibrators and corrected from their diameter estimated from color indexes. The observations were made in the $\mathrm{L}$ band, from 3.1 to $3.9 \mu \mathrm{m}$ with a frame time of DIT=75 ms. We see that the response is much more sensitive to $\tau_{0}$ than to seeing and becomes little sensitive to atmospheric changes for $\tau_{0}>5 \mathrm{~ms} \simeq$ DIT $/ 15$.

So, the first impact of seeing changes is a variation of the coherent flux due to a loss in instrumental visibility that can exceed a factor 2 when we move from "fair" conditions $\left(\tau_{0}>4 \mathrm{~ms}\right)$ to bad conditions $\left(\tau_{0}<3 \mathrm{~ms}\right)$. All the limits given here are in coherent flux. The users must consider these changes in coherent flux and hence in performances when they define the seeing conditions required for their observations.

\subsection{Broad Band Calibration errors due to seeing changes}

To evaluate the calibration errors due to seeing changes we have analyzed the so-called "time transfer functions" that show the variation of the broad-band measurements on bright calibrators as a function of time, as illustrated in figure 4 . Then, we mimic a "snapshot mode" calibration-science-calibration sequence, i.e. in a cal1-cal2-cal3 sequence we calibrate cal2 with an average from call and cal2 and we make statistics on the accuracy of the result. Alternatively, we mimic an "imaging mode" calibration based on the rms difference between each calibrator and a global fit through all the calibrators of a night. The two methods give the very similar results that are summarized in table 3 . 

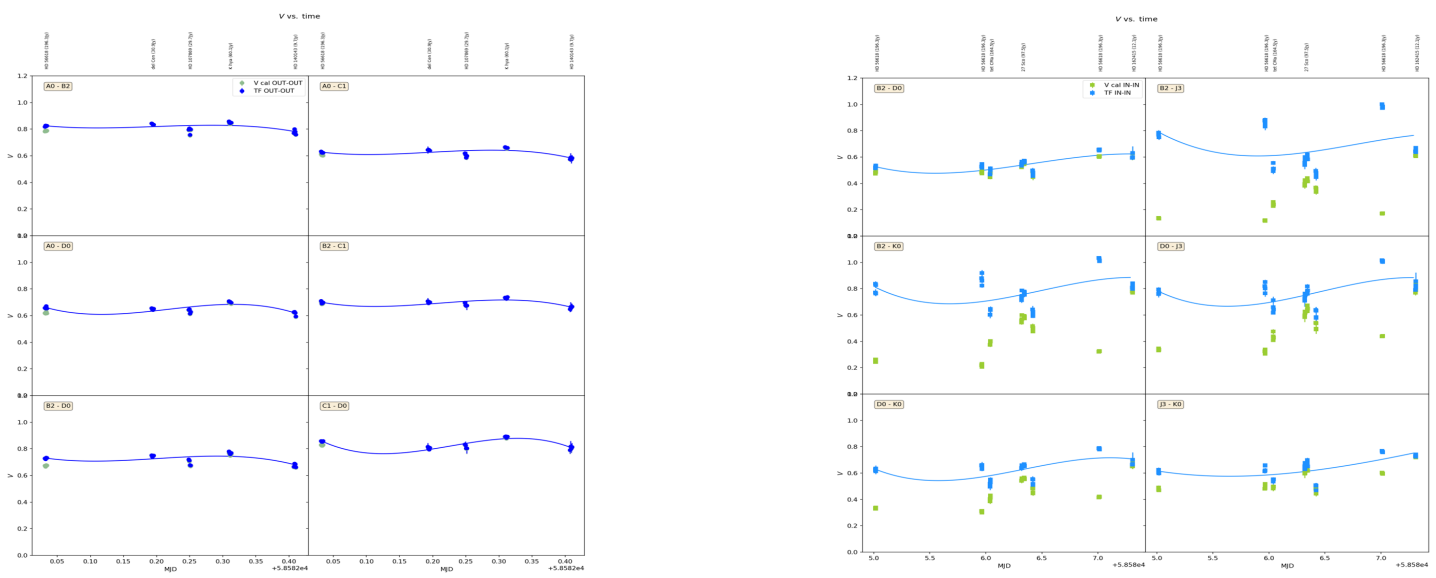

Figure 4. Example of visibility "transfer function" vs time, in L band at low resolution with ATs, for a good night (left, seeing=0.6as) and a bad night (right, seeing=1as). The blue dots are for calibrator visibility corrected from the calibrator diameter. The green dots are for raw visibility measurements. The rms of the difference between the measures and the fit is an estimate of the broad band calibration error.

Table 3. Broad band calibration errors on visibility and closure phase as a function of seeing conditions, for the standard frame times of MATISSE, $75 \mathrm{~ms}$ in L only, $111 \mathrm{~ms}$ in L\&M and a 10 frames modulations cycle of $240 \mathrm{~ms}$ in N. Note that the closure phase numbers are given using the BCD calibration. A calibration using only external calibrators yields broad band closure phase errors between $1^{\circ}$ and $2^{\circ}$.

\begin{tabular}{|c|c|c|c|c|c|c|c|}
\hline \multirow{2}{*}{\multicolumn{2}{|c|}{ Seeing conditions }} & \multicolumn{2}{|c|}{$\mathbf{L}$} & \multicolumn{2}{|c|}{$\mathbf{M}$} & \multicolumn{2}{|c|}{$\mathbf{N}$} \\
\hline & & \multicolumn{2}{|c|}{$\mathrm{DIT}=75 \mathrm{~ms}$} & \multicolumn{2}{|c|}{$\mathrm{DIT}=111 \mathrm{~ms}$} & \multicolumn{2}{|c|}{ DIT. $\mathrm{N}_{\mathrm{mod}}=240 \mathrm{~ms}$} \\
\hline$\tau_{0}(\mathrm{~ms})$ & Seeing (as) & Visibility & Closure Phase & Visibility & Closure Phase & Visibility & Closure Phase \\
\hline $3.2 \pm 0.5$ & $0.96 \pm 0.1$ & 0.08 & $0.30^{\circ}$ & 0.05 & $0.25^{\circ}$ & 0.045 & $1.75^{\circ}$ \\
\hline $6.8 \pm 0.5$ & $0.74 \pm 0.1$ & 0.02 & $0.26^{\circ}$ & 0.020 & $0.24^{\circ}$ & 0.02 & $0.49^{\circ}$ \\
\hline $7.5 \pm 0.5$ & $0.56 \pm 0.1$ & 0.02 & $0.16^{\circ}$ & 0.015 & $0.15^{\circ}$ & $0 . .015$ & $0.29^{\circ}$ \\
\hline
\end{tabular}

\subsection{Broad band photometric calibration errors.}

To estimate the visibility from the coherent flux measurements, we need to separate the source and background contribution to the photometric measures. For all sources in $\mathrm{N}$ and $\mathrm{M}$ and for faint sources in $\mathrm{L}$ this needs telescope chopping. The broad band photometric errors produce an additional source flux dependent error. We have estimated it from statistics of the measured photometry for bins of calibrator with similar magnitudes. On ATs we have a data base allowing reliable statistics. On UTs, we dot have enough data and we assume that the photometric errors are the same as on UTs with of course a different conversion into source equivalent flux. From MIDI time we know that this is a pessimistic and conservative estimate for UTs that are less sensitive to chopping defects than the ATs. The actual measurements made with UTs confirm that we are quite certainly slightly overestimating the photometric noise with UTs.

The estimation of the noise on the photometric estimates due to the background fluctuations have been based on a statistical analysis of the sky frames obtained from telescope chopping, for the full $\mathrm{L}$ and $\mathrm{M}$ bands and $1 \mu \mathrm{m}$ sub-bands in N.

Table 4. Broad band photometric errors given in equivalent source flux in Jy for all spectral bands (at $9 \mu \mathrm{m}$ in $\mathrm{N}$ ). The UTs values are deduced from the AT values using the measured UT/AT flux ratios.

\begin{tabular}{|cccc|}
\hline & $\mathbf{L}$ & $\mathbf{M}$ & $\mathbf{N}$ \\
$\mathbf{A T}$ & $0.11 \mathrm{Jy}$ & $0.19 \mathrm{Jy}$ & $2.3 \mathrm{Jy}$ \\
$\mathbf{U T}$ & $0.008 \mathrm{Jy}$ & $0.016 \mathrm{Jy}$ & $0.08 \mathrm{Jy}$ \\
\hline
\end{tabular}

We found that the photometric noise in the N band on AT3 (that is always feeding the same VLTI beam and set of relay optics labelled "IP5") we found a photometric noise very often higher than the number in table 4 that is the one for the 
three other telescopes. On ATs the rms photometric error is of the order of $5 \mathrm{Jy}$. We found that replacing the ATs photometry by a combination of the other 3 telescopes photometry substantially improves the calibration of the measures. This is an option in the data reduction pipeline that we have used systematically for all performances reported here. The cause for this extra noise on AT3 is under investigation with the VLTI team, without conclusion so far.

The analysis of photometric noise also showed that the variation in time, between successive photometric measurements, is larger than the variations between beams or BCD configuration, when the constant average differences have been corrected. This opens the way for a reduction of the photometric sequence, where we could use 3 photometric exposures in $\mathrm{N}$ instead of 8 , to be tested with existing data and in the last commissioning run.

All the numbers given in table 4 are for observations with chopping. In $\mathrm{N}$ and $\mathrm{M}$, chopping is mandatory. In L, it becomes critical for faint stars. For $\mathrm{L}>25 \mathrm{Jy}$, the background measurement with a single separated sky exposure is sufficient for all wavelengths. At $3 \mu \mathrm{m}$, chopping becomes critical only for targets fainter than $4 \mathrm{Jy}$. The limiting wavelength $\lambda_{S}$ and the flux level $L_{S}$ making chopping necessary vary nearly linearly between these two limits. In L band, visibility measurements require chopping if the target has a flux

$$
L_{S} \lesssim 18\left[\lambda_{S}(\text { in } \mu m)-3\right]+4 J y
$$

As the chopped $\mathrm{L}$ and $\mathrm{M}$ band observations are obtained during the so-called $\mathrm{N}$ band photometry, this is an argument to avoid reducing too much these exposures.

\subsection{Bias on the coherent flux}

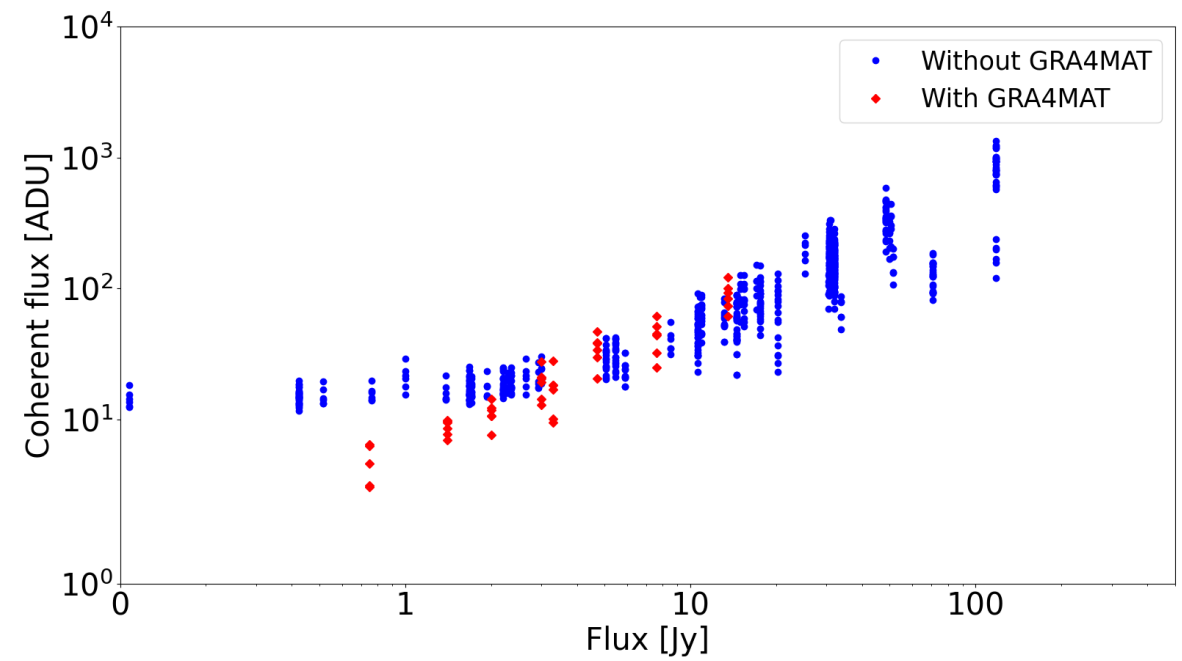

Figure 5. Measured coherent flux as a function of the source flux. The measures are made for $1 \mathrm{mn}$ exposures and averaged over a $1 \mu \mathrm{m}$ spectral band. The blue dots are for a processing with phase delay estimates from the $\mathrm{N}$ band itself. They show a bias on the coherent flux for targets fainter than $\sim 5 \mathrm{Jy}$. The red dots are for a processing with phase delays deduced from the GRA4Mat measures in the $\mathrm{K}$ band. Then the bias, if any, is below $1 \mathrm{Jy}$.

In N band, below typically 20 Jy on ATs, the SNR on the coherent flux per spectral channel and coherence time becomes comparable or lower to one. Then, adding the squared modulus of the coherent flux becomes very inefficient as indicated by equation (14) in section 3.2. It is then necessary to perform a coherent integration of the complex coherent flux beyond the coherence time. This is done by an estimate of the global phase delay in each frame that is then corrected before direct addition. This is equivalent to shifting all fringes in individual frames to the same position before adding them in a long exposure. This improves quite substantially the precision for all measures. However, errors on the estimation of the phase delay in each frame yield a bias on the coherent flux that stops to decrease proportionally to the source flux. When the phase delay is estimated on the $\mathrm{N}$ band data of the source itself, this bias limits to $\sim 5 \mathrm{Jy}$ at $8.5 \mu \mathrm{m}(\sim 8 \mathrm{Jy}$ at $11 \mu \mathrm{m})$ the possibility to obtain reliable coherent fluxes as shown by figure 5 . 


\section{GLOBAL PERFORMANCES}

\subsection{Method}

Figure 6 illustrates the global performance estimate that combines the different contributions to the MATISSE measurement errors. The left panels show the fundamental noise measures, fitted by the computed prediction for the visibility, the closure phase and the differential phase. For the differential phase, we measure directly the precision on the fundamental noise plot, and this gives the precision on the differential phase between one channel and a nearby reference channel or a broad reference channel if this has been correctly corrected from chromatic OPD. The actual differential phase accuracy in that latest case remains an open issue as explained at the end of section 2.2 around equation (9).

LOW L AT
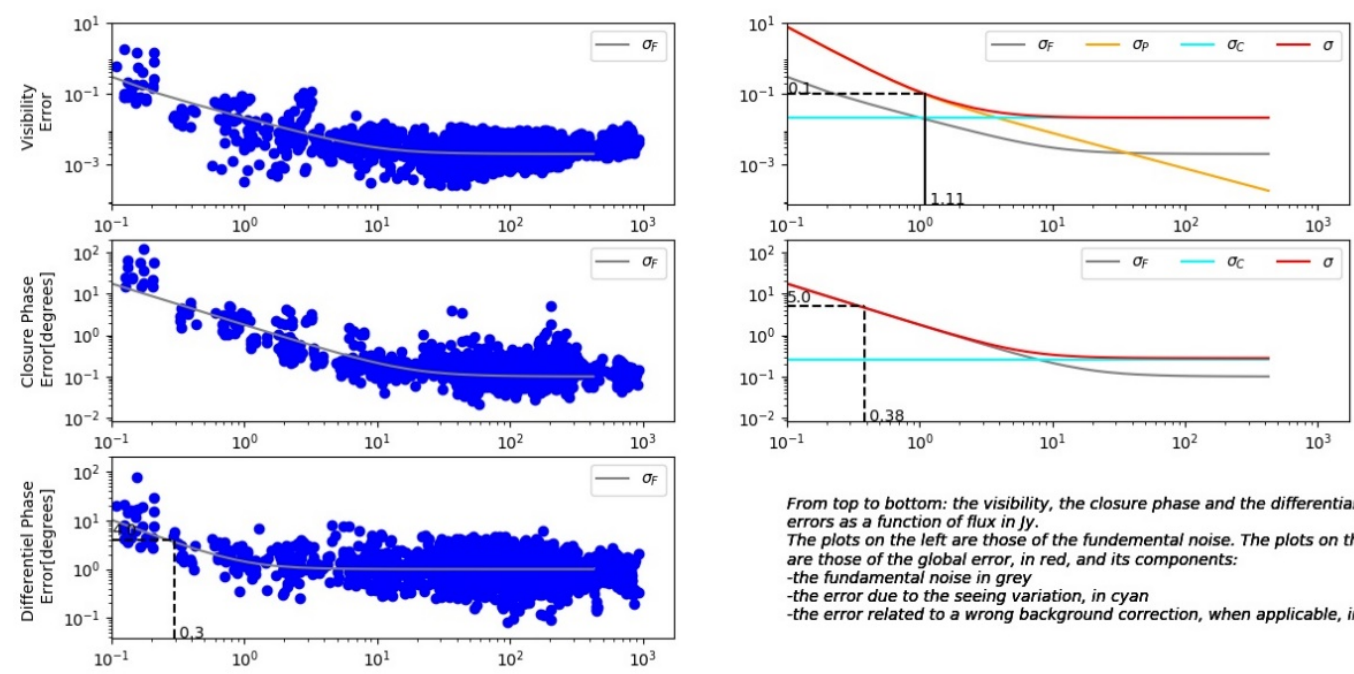

From top to bottom: the visibility, the closure phase and the differential phase errors as a function of fiux in $\mathrm{yy}$. The plots on the left are those of the fundemental noise. The plots on the right are those of the global error, in red, and its components: the error due to the seeing varitic

Figure 6. Illustration of the general performance of MATISSE in LR-L with ATs. In the left column we plot the measured fundamental noise per spectral channel (blue dots) as a function of the source coherent flux. The grey line shows the value of the computed precision that goes through the median of the measures. Visibility are on top (in visibility units), Closure Phase is in the middle and Differential Phase is on bottom (both in degrees). The coherent flux is in Jy. The top right panel shows the visibility fundamental noise standard deviation (grey), the flux dependent broad band photometric error (yellow) and the broad band calibration error due to seeing changes that is independent from the flux (cyan) as well as their combination (red) by quadradic addition assuming these are independent variables.

For the closure phase, we add the variance of the fundamental noise per channel to the broad band calibration variance in table (3) deduced from the time transfer function of the measure as described in section3.5. Then the plot of these combined variances is used to find the coherent flux that yields a closure phase accuracy per spectral channel better than $5^{\circ}$ in $50 \%$ of the cases.

For the absolute visibility we add the variances of the fundamental noise errors, the seeing broad band calibration error and the photometric broad band calibration error and use the plot of the combined variance to find the coherent flux that yields a visibility accuracy per spectral channel better than 0.1 in $50 \%$ of the cases. This has been done for UTs and ATs and for all spectral set-ups.

\subsection{Validation}

To check the overall validity, we have plotted the broadband measured visibility as a function of the flux, as illustrated, as an example, in LR-N in figure 7. We see clearly the flux independent regime above $30 \mathrm{Jy}$ in that mode. The rms value in that regime is between 0.02 and 0.03 and gives the calibration error for a science target calibrated by a calibrator obtained at any moment, including in a different night. In $\mathrm{N}$ band this is possible when we use the short $20 \mathrm{~ms}$ frames individually (on bright enough sources) because that frame time is much shorter than the coherence time in N. Globally and except at very low fluxes (which are below the limit that we offer for that observation and that mode) almost all points are within the $\pm 1 \sigma$ interval of our global performance estimation. 

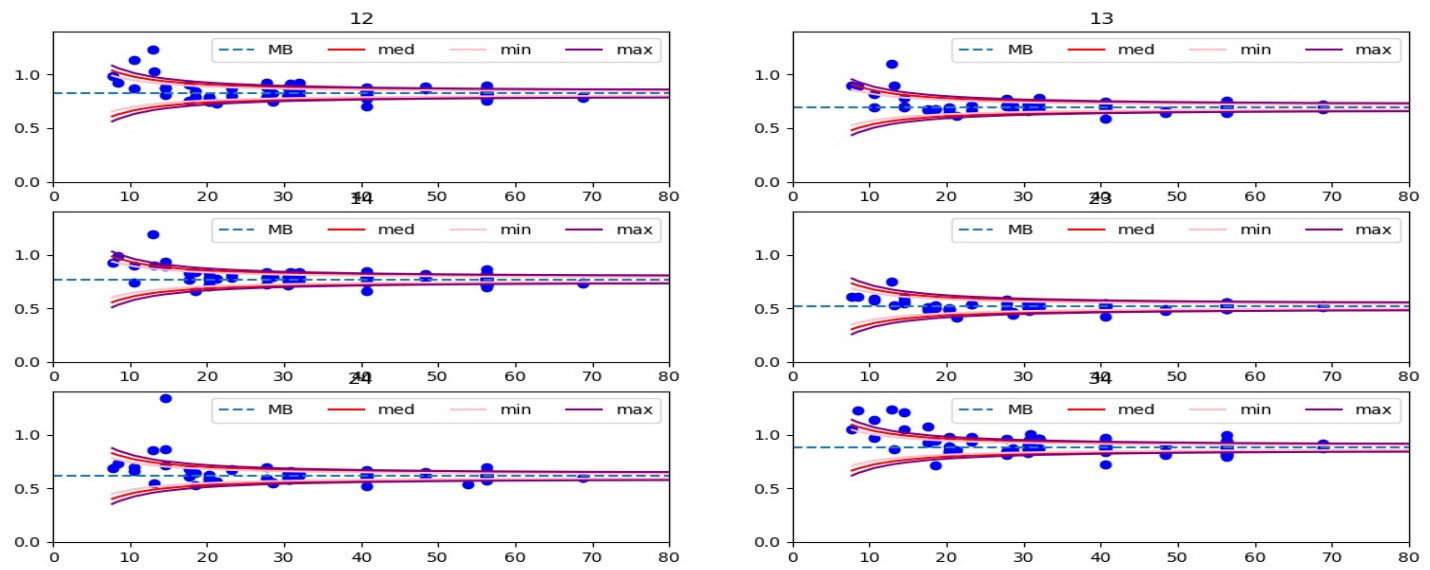

Figure 7. Measured instrument + atmosphere visibility as a function of the source flux (blue dots), here in LR-N averaged between 8 and $9 \mu \mathrm{m}$, with the ATs. The dashed horizontal line in the mean instrument visibility that ranges from 0.6 to 0.9 according to the baseline. The red lines are the mean \pm 1 median sigma. The yellow and brown lines correspond to the best and the poorest observing conditions. The limiting coherent flux for a 0.1 visibility accuracy is 17 Jy for that mode and spectral band. Above that limit, almost all experimental points are within $\pm 1 \sigma$.

\subsection{The current performances of MATISSE standalone}

Table 5. MATISSE sensitivity limits defined has the lowest flux in Jy allowing to achieve a visibility accuracy of 0.1 , a closure phase accuracy of $5^{\circ}$, a differential phase precision of $4^{\circ}$ and a coherent flux SNR of 10 .

\begin{tabular}{|c|c|c|c|c|c|c|c|c|c|c|c|c|}
\hline \multirow[b]{2}{*}{ Telescope } & \multirow[b]{2}{*}{ Resolution } & \multicolumn{3}{|c|}{ Visibility } & \multicolumn{3}{|c|}{ Closure Phase } & \multicolumn{3}{|c|}{ Differential Phase } & \multicolumn{2}{|c|}{ CF bias limit } \\
\hline & & $\mathbf{L}$ & $\mathbf{M}$ & $\mathbf{N}$ & $\mathbf{L}$ & $\mathbf{M}$ & $\mathbf{N}$ & $\mathbf{L}$ & $\mathbf{M}$ & $\mathbf{N}$ & N1 & N2 \\
\hline \multirow{3}{*}{ ATs } & Low & 1.1 & 2.1 & 16.8 & 0.4 & 1.9 & 9.4 & 0.3 & 1.1 & 2.9 & 5 & 8 \\
\hline & Medium & 3.8 & 16 & - & 3.3 & 15.6 & - & 2.4 & 11 & - & - & - \\
\hline & High & 20.1 & - & 30.3 & 14.7 & - & 29.9 & 10.8 & - & 25.3 & 5 & 8 \\
\hline \multirow{3}{*}{ UTs } & Low & 0.3 & 0.4 & 0.9 & 0.07 & 0.2 & 0.3 & 0.06 & 0.15 & 0.07 & 0.3 & 0.5 \\
\hline & Medium & 1.1 & 1.1 & - & 0.8 & 0.9 & - & 0.6 & 0.7 & - & - & - \\
\hline & High & 2.4 & - & 1.6 & 1.7 & - & 1.5 & 1.2 & - & 1.1 & 0.3 & 0.5 \\
\hline
\end{tabular}

The table 5 summarizes the current flux limits to obtain, per spectral channel and per mn of observation, a visibility accuracy better than 0.1 , a closure phase accuracy better than $5^{\circ}$ and a differential phase precision better than $4^{\circ}$. For all modes but for the LR in $\mathrm{N}$, the differential phase precision of $4^{\circ}$ correspond to a coherent flux SNR of 10 , according to equation (15) in section 3.2. In LR-N we have also to consider the coherent flux bias described in section 3.7 that sets a lower flux for its use. The values given in table 5 are for good and fair seeing conditions, i.e. seeing $<0.9$ arcseconds and $\tau_{0}>4 \mathrm{~ms}$. When the seeing conditions are poor $\left(\tau_{0}<3 \mathrm{~ms}\right)$, the coherent flux can lose up to 1 magnitude and all limits in table 5 move up by typically $2 \mathrm{Jy}$.

The limits in table 5 are given for the center of the bands as described in table 6 . Near the band edges the measurement errors can be larger, particularly on the differential phase that also partially contaminates the closure phase, through crosstalks between baselines, and the visibility through the inclination of the fringes.

Table 6. Spectral band used to define the measurement performances given in table 5.

\begin{tabular}{|c|c|c|c|c|}
\hline resolution & $\mathbf{L}$ & $\mathbf{M}$ & $\mathbf{N} \& \mathbf{~ N 1}$ & N2 \\
\hline Low & $3.5 \pm 0.3 \mu \mathrm{m}$ & $4.75 \pm 0.2 \mu \mathrm{m}$ & $8.5 \pm 0.5 \mu \mathrm{m}$ & $10.5 \pm 0.5 \mu \mathrm{m}$ \\
\hline Medium & 0.2 in $[3.1-4.2] \mu \mathrm{m}$ & 0.2 in $[4.5-5] \mu \mathrm{m}$ & Does not exist & Does not exist \\
\hline High & 0.1 in $[3.8-4.2] \mu \mathrm{m}$ & Does not exist & In [8-9] $\mu \mathrm{m}$ & In $[10-11] \mu \mathrm{m}$ \\
\hline
\end{tabular}




\section{THE GRA4MAT AND VHR EXTENSIONS}

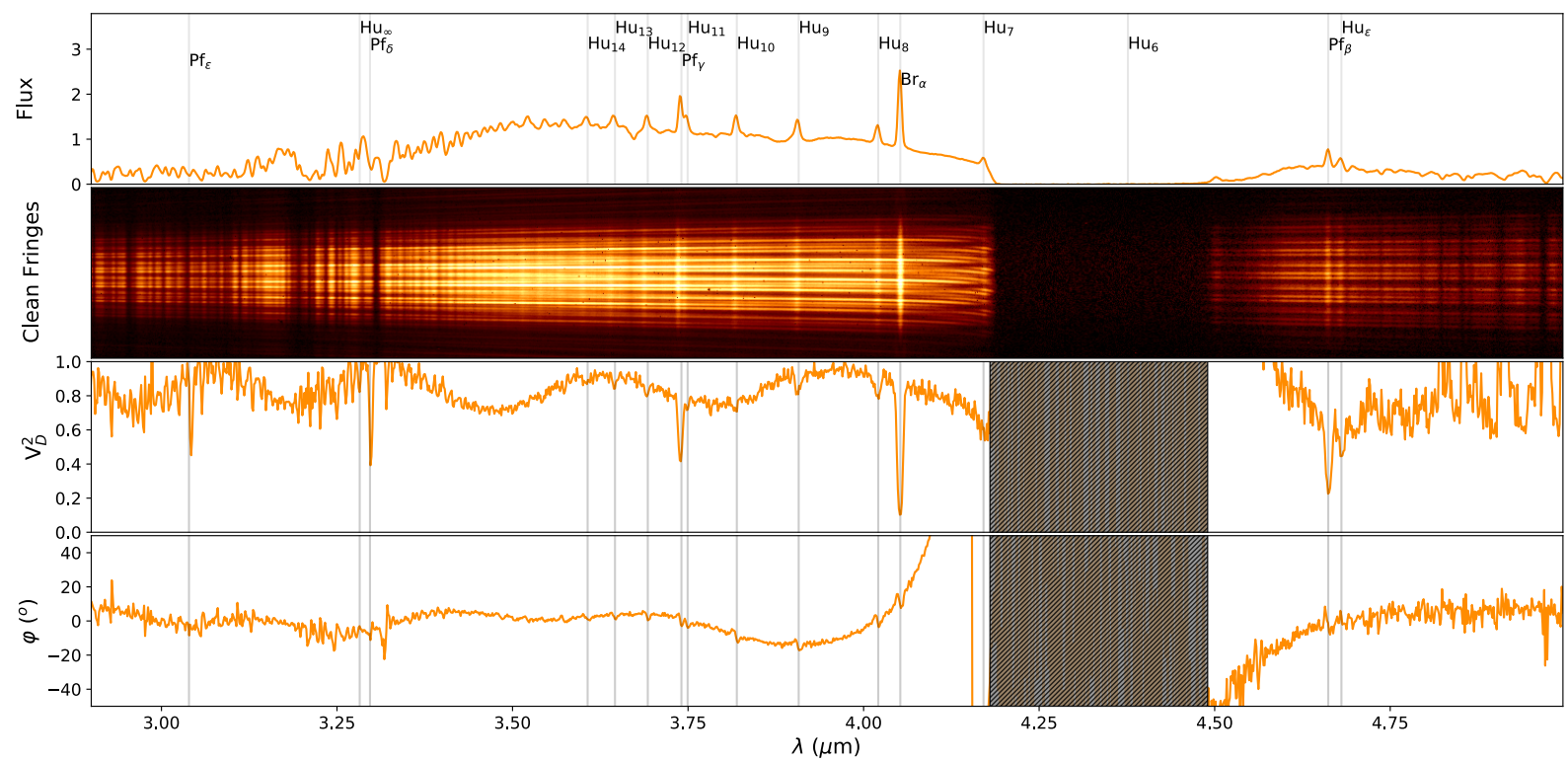

Figure 8. MATISSE MR-LM observations in the GRA4MAT mode with a fringe stabilization with the Gravity Fringe Tracker. The image in the second line shows the interferometric channel of MATISSE with a $10 \mathrm{~s}$ frame time with straight and stable high contrast fringes from 3.9 to $4.9 \mu \mathrm{m}$ and a collection of bright emission lines and darks telluric absorption lines. The upper plot is the source spectrum showing a rich collection of hydrogen emission lines in Bra and the Humphrey and Pfund series. The second to bottom plot show the measured visibility and the bottom plot shows the differential phase.

In late 2019 we started commissioning simultaneously the Very High-Resolution mode of MATISSE (R 3400 in L and M, from 3.85 to $4.9 \mu \mathrm{m}$ ) and the GRA4MAT mode, that is the use of the GRAvity fringe tracker FOR the stabilization of MATISSE fringes. The GRAVITY Fringe Tracker is operated in the $\mathrm{K}$ band and that sets specific limits for the coherent magnitude in $\mathrm{K}$ that are given in table 7.

Table 7. $\mathrm{K}$ band coherent magnitude limit for GRA4MAT operation. Note that the seeing bins in that table are the standard ESO ones, while the seeing bins used in the MATISSE tables (3) and (5) are from actual but more limited statistics on our data.

\begin{tabular}{|cccc|}
\hline Conditions & Good $(\mathrm{T} \leq 10 \%)$ & Fair $(\mathrm{T} \leq 50 \%)$ & Poor $(\mathrm{T} \leq 85 \%)$ \\
Seeing and $\tau_{0}$ & $\mathrm{Sg} \leq 0.6 " ; \tau_{0}>5.2 \mathrm{~ms}$ & $\mathrm{Sg} \leq 1.0 " ; \tau_{0}>3.2 \mathrm{~ms}$ & $\mathrm{Sg} \leq 1.4 " ; \tau_{0}>1.6 \mathrm{~ms}$ \\
K coherent limit & 8.5 & 7.5 & 6.5 \\
\hline
\end{tabular}

Without GRA4MAT, the VHR mode gives access to $0.025 \mu \mathrm{m}$ spectral windows for sources brighter than $40 \mathrm{Jy}$ in $\mathrm{L}$ and $55 \mathrm{Jy}$ in M with ATs. This is a very limited range of application, and the VHR mode on ATs is and will remain offered only in association with fringe tracking with GRA4MAT.

\section{- GRA4MAT gives access to the full spectral bands in all spectral resolutions}

Within the K band limits in table (7) GRA4MAT allows long exposures in L\&M that enable reading the full detector and hence accessing simultaneously the full MATISSE spectral coverage as illustrated in figure (8). That figure displays one $10 \mathrm{~s}$ frame on the Be binary star $\delta$ Cen, which shows the very rich information that can be extracted from a single shot broad band observation at medium or high spectral resolution. We can observe simultaneously many emission lines, each showing differential visibility and differential phase signatures that constrain, respectively, the size, the kinematics and the asymmetry of the gas envelope. The small oscillations of the visibility in the continuum reveal the binarity of $\delta$ Cen. This figure also illustrates the strong effect of chromatic OPD near the band limits. This has a strong impact on the differential phase and a careful modeling and calibration of that chromatic OPD will be needed to push the continuum differential 
phase accuracy below the current $1^{\circ}$ accuracy. This chromatic OPD has also an attenuated but real impact on the visibility and closure phase that limits the current dynamics of MATISSE to about 300. The analysis and calibration of the differential phase should allow modeling and correcting these effects and hence increasing the capability of MATISSE to characterize fainter companions. We can also analyze the many telluric lines that can be seen between 3 and $3.5 \mu \mathrm{m}$ and between 4.7 and $5 \mu \mathrm{m}$, which have a hidden impact on the accuracy of broad band measures at lower resolutions.

\section{- GRA4MAT reduces the broad band calibration errors}

GRA4MAT very strongly reduces the piston jitter seen by MATISSE and hence strongly stabilizes the instrument visibility, that becomes nearly unsensitive to seeing, within the operation limits of GRA4MAT. The gain in instrument calibration has not been fully investigated but it should at least make the numbers for the best seeing in table (3) of section 3.5 valid for all conditions that allow using GRA4MAT.

\section{- GRA4MAT improves the sensitivity limits for the higher spectral resolution modes}

The possibility to use longer frame times (DIT for Detector Integration Time) yields sensitivity gains that have been measured with results summarized in table 8 below.

Table 8. Limits in Jy to achieve differential phase precision of $4^{\circ}$ and a coherent flux $\mathrm{SNR}=10$ and a closure phase accuracy of $5^{\circ}$, per spectral channel and per $1 \mathrm{mn}$ exposure.

\begin{tabular}{|c|c|c|c|c|c|c|c|c|}
\hline \multicolumn{2}{|l|}{ AT } & \multicolumn{3}{|c|}{ Closure Phase } & \multicolumn{3}{|c|}{ Differential Phase \& Coherent Flux } & \multirow{2}{*}{$\begin{array}{c}\text { CF bias limit } \\
\mathrm{N}\end{array}$} \\
\hline Resolution & DIT & $\mathrm{L}$ & M & $\mathrm{N}$ & $\mathrm{L}$ & M & $\mathrm{N}$ & \\
\hline Low & $1 \mathrm{~s}$ & 0.25 & 1 & $\sim 4.1 \mathrm{tbc}$ & 0.17 & 0.7 & 2.9 & $<2.9$ \\
\hline Medium & $10 \mathrm{~s}$ & 1.5 & 15.6 & -- & 1 & 11 & -- & -- \\
\hline High & $10 \mathrm{~s}$ & 3 & -- & 29.9 & 2 & -- & 25.3 & $\ll 25$ \\
\hline Very High & $10 \mathrm{~s}$ & 25 & 25 & -- & 20 & 17 & -- & -- \\
\hline
\end{tabular}

No gain in sensitivity for the visibility, at least for the time being. In $\mathrm{N}$ and $\mathrm{M}$ the visibility limit is dominated by the precision of the photometric measures that cannot not improved by GRA4MAT. Moreover, the first implementation of GRA4MAT did not permit chopping while fringe tracking. As chopping is necessary for accurate visibility in $\mathrm{M}$ and in faint L sources, the provisional procedure currently offered combines MATISSE standalone observations at low resolution with chopping and without GRA4MAT, that still give the best absolute visibility measures, and GRA4MAT observations at higher spectral resolution that give the performances in table 8 for the other measures. The operation of GRA4MAT with chopping is possible since March 2020, but the covid-19 crisis delayed its full commissioning. It should be offered after April 2021.

No gain in sensitivity in MR-M and HR-N (medium resolution in M and high-resolution in N), because we are in a regime completely dominated by the background photon noise and hence unsensitive to the frame time for a constant total exposure time.

Modest gain in LR-L, MR-L and LR-M (low and medium resolution L and in low resolution in M), because we are in a regime nearly dominated by the source or the background photon noise, but partially sensitive to detector read-out-noise.

Very strong gain in HR-L and VHR-LM (high resolution L and in very high resolution in L and M), because we are completely dominated by read-out-noise without GRA4MAT. The VHR mode does not really make sense without GRA4MAT.

\section{- GRA4MAT improves the sensitivity for coherent flux and closure phase in LR-N}

In the $\mathrm{N}$ band GRA4MAT allows coherent integration of frames [13] without bias. The phase delay and group delay in the $\mathrm{K}$ band given by the GFT allow computing the chromatic phase difference between $\mathrm{K}$ and $\mathrm{N}$ [14] and then measuring the phase delay in $\mathrm{N}$ with an accuracy better than $0.2 \mathrm{rad}$. This makes the coherent flux bias lower than the fundamental noise limits, as illustrated in figure 5. The $\mathrm{SNR}=10$ coherent flux limit with MATISSE is then identical to the differential phase precision limit $=4^{\circ}$ given in table 5, i.e. $2.9 \mathrm{Jy}$ with ATs. In addition, that coherent flux integration drives us in the $\sigma_{\varphi_{i j 1}}<$ $1 \mathrm{rad}$ regime discussed in section 3.2 and the closure phase precision should be given by equation (16), i.e. $\sigma_{\psi}=$ $\sigma_{\varphi} \sqrt{3} \sim 4.1$ although this still needs to be confirmed experimentally. 


\section{- MATISSE is sensitive to fringe jumps in the GRAVITY Fringe Tracker (GFT)}

The GFT can produce one $\lambda(\lambda \sim 2.2 \mu \mathrm{m})$ fringes that have little impact in the $\mathrm{K}$ band but strongly affect the visibility at the MATISSE wavelength where they represent a fringe shift of a fraction of wavelength. We are developing software to detect the fringe jumps and eliminate the affected MATISSE frames. The number of fringe jumps will depend of the source flux in $\mathrm{K}$ and it will limit the frame time usable in MATISSE. With ATs it seems reasonable to use DIT=10s down to the GRAVITY $\mathrm{K}$ band limits. This is much shorter that the frame times used in GRAVITY that can reach 2 minutes. The very preliminary test made with UTs indicate that the MATISSE maximum frame time might be limited to a very small number of seconds because of the higher fringe jump rate due to the relative instability of the MACAO adaptive optics. This fringe jumps issue will be a critical parameter to investigate in the last and pending GRA4MAT commissioning run.

\section{IMAGING WITH MATISSE}

In December 2018 we performed an "Imaging Commissioning" run with MATISSE. We used the standard configurations with ATs (small, medium and large) and 4 intermediate configurations corresponding to the relocation of only two ATs in the evolution between the standard configurations. We spent typically two nights per configuration and concentrated on three targets with their calibrators for which we obtained the maximum possible $\mathrm{u}-\mathrm{v}$ coverage. The run allowed to fit in partial observations of 3 more targets. The run was fully successful and demonstrated the imaging capability of MATISSE and the specific image reconstruction tool IRBis that has been developed for it [15]. Remarkably, we have been able to reconstruct several images through the run with the data processed by the standard MATISSE pipeline as it was in late 2018. This was achieved with the MATISSE image reconstruction package and also by the reference image reconstruction tool MiRA [16]. This is illustrated by figure 9 that shows the image of the HeBe star FS CMa in the L and N bands obtained at the $11^{\text {th }}$ day of our 14 days run, with 5 configurations (out of the final 7). These images have been overplotted with a previous PIONIER image in the $\mathrm{H}$ band [17], which helped to validate our image reconstruction. This image reconstruction has been very substantially improved in resolution, accuracy and dynamical range after the end of the run with the full u$\mathrm{v}$ coverage and a more careful data calibration and selection, but the fundamental shape of the image and its key characteristics already clearly appeared during the run in the reconstruction obtained from partial data. This was a very strong test of the validity of our automatic pipeline and it showed that the completeness of image reconstruction data can be checked during an imaging run by people having a correct expertise in the use of image reconstruction software in interferometry. Another important lesson from this run is the possibility to change configuration every two days if we move no more than two telescopes at once. This is permitted if we use the intermediate configuration that appeared extremely valuable in speeding up and improving the $u-v$ coverage.

\section{CONCLUSION}

The performances of MATISSE described in this paper have been established on a solid statistical basis, in particular with ATs, and we consider the numbers given here as quite reliable. The high calibration accuracy achieved almost always in closure phases and on visibility in fair and good seeing conditions will be strongly constraining on the adjustment of models. A model fit that is more than $3 \sigma$ away from our combined error bars should really be considered as problematic.

In our performance analysis we have clearly separated the fundamental noise terms, that are nearly independent between spectral channels, from the broad band calibration errors. We would like to insist on the importance to treat the errors per spectral channel and the broad band errors specifically in model fitting and in image reconstruction. It is important to note that the error bars produced "on-line" by the MATISSE data reduction software are based on the temporal dispersion between sub exposures. In addition to some specific biases in coherent integration modes (where the SNR is not proportional to the square root of the exposure time), these error bars merge the fundamental noise by spectral channel and the broad band variation at the scale of the exposure and ignore the calibration errors due to broad band variations over a calibration cycle. The python tools that we use in our performance analysis can be given to any user. The "Exposure Time Calculator" in the ASPRO JMMC preparation tool of interferometric observations is being transformed to include our different contributions to MATISSE measurement precision and accuracy and some talks are in progress with ESO to find out this information can be efficiently given to all users. For help and more information please visit the JMMC webpage: www.jmmc.fr/SUV. 


\section{FS CMa infrared composite image}

\section{PIONIER H band}

MATISSE L band MATISSE $\mathbf{N}$ band
10 mas

3 AU

MATISSE images: MATISSE commissioning team 14Dec2018; Graphics: A. Meilland; PIONIER image: KIuska 2014IAUS..299..117K
HD45677, MATISSE, 60.A-9257(E)

-> 2018-12-12T๑8:46:21.4466

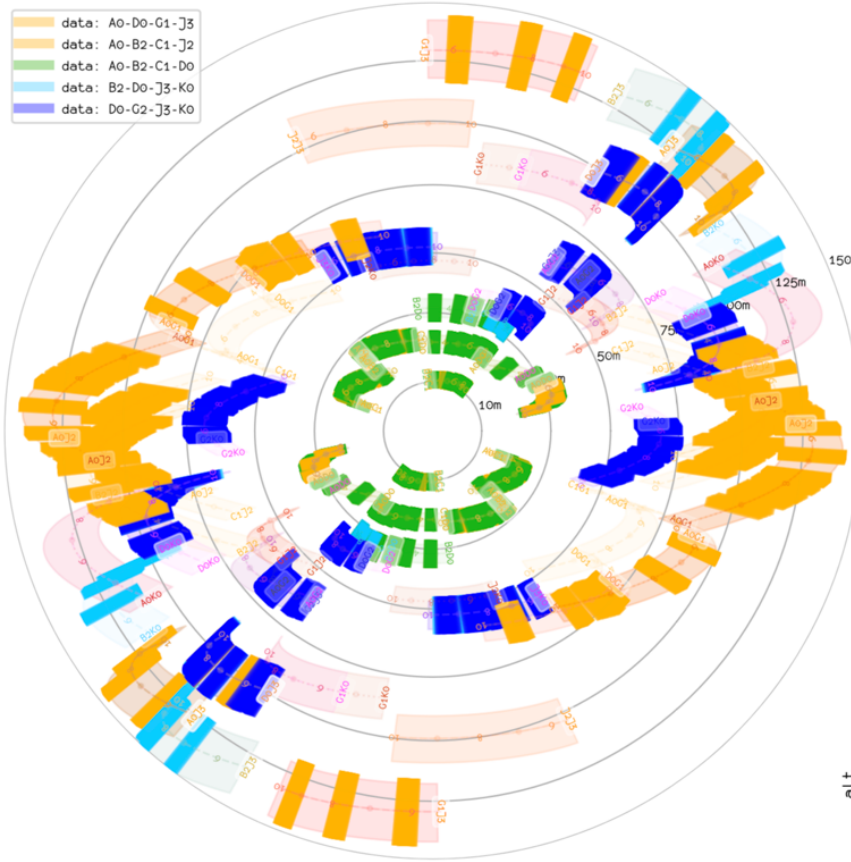

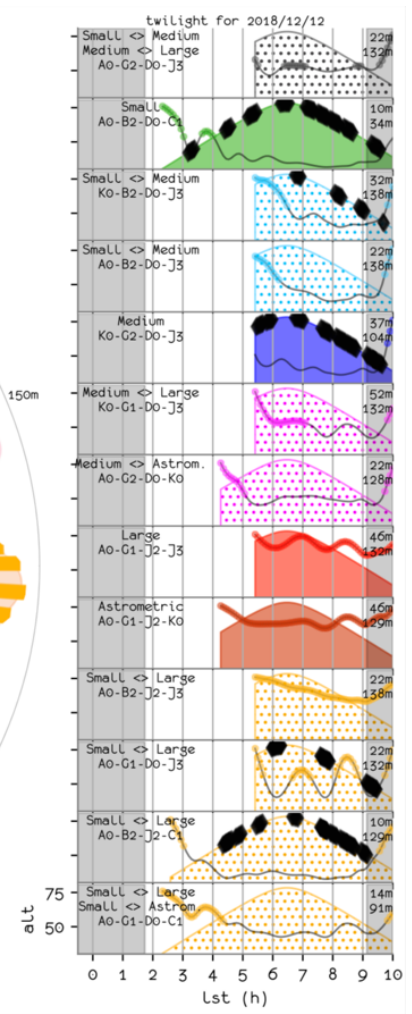

Figure 9. Composite image of the dust around FS CMa (top) obtained during our December 2018 "imaging commissioning run". We have overplotted the previous PIONIER H band image [17] and the MATISSE L and $\mathrm{N}$ band images obtained when our December 2018 imaging commissioning run was completed at $70 \%$. The bottom image shows the $\mathrm{u}-\mathrm{v}$ coverage used for that reconstruction. In light salmon, the maximum potential $\mathrm{u}-\mathrm{v}$ coverage and in bold colors the u-v coverage actually achieved at that moment and used in the reconstructed image displayed in the top figure. In green and blue the standard "small" and "medium" VLTI configurations. In cyan and yellowish the intermediate configurations between "medium and small" and between "small and large". 
During the December 2018 "imaging commissioning run" we have been able to obtain images during the run itself. Of course the images obtained later after a careful data processing and calibration were of much higher quality but it is quite remarkable that, with some good expertise of MATISSE image reconstruction tools (that can be acquired in a couple of weeks), it is possible to evaluate the imaging quality of the data and for example the completeness of the u-v coverage in the hours that follow the end of each night.

One of the specifications of MATISSE was that it should achieve in the $\mathrm{N}$ band the same data quality than the firstgeneration instrument MIDI. As showed by the numbers given in this paper and by several science examples in [5] and [8] this has been fully achieved, for each baseline for visibility and coherent flux, with the advantage that MATISSE obtains 6 baselines at once and gives closure phases for the first time in the L,M and N bands. The sensitivity of MATISSE in the $\mathrm{L}$ band will be a game changer for the study of AGNs as it strongly increases the number of observable candidates. The simultaneous observations in $\mathrm{L}, \mathrm{M}$ and $\mathrm{N}$ are a decisive constraint on the source temperature and composition. Because of the higher sensitivity of the $\mathrm{L}$ band, this is possible on almost all the targets previously observed by MIDI with the huge additional advantage offered by image reconstruction.

GRA4MAT opens the possibility to do images at medium and high spectral resolution with the ATs, another feature decisive for the mineralogy of YSO disks where grains or molecules with specific spectral signature will be precisely located in the disk.

The commissioning of MATISSE with UTs has been interrupted by the covid-19 shutdown. It is particularly incomplete in the case of GRA4MAT. It should be executed as soon as the covid-19 crisis makes it possible. The preliminary investigations of this mode in March 2020, just before the shutdown of Paranal showed that GRA4MAT with UTs will work but probably with a smaller gain than with ATs, in particular because of the instability of MACAO that is likely to limit the frame time to $1 \mathrm{~s}$.

Three points need deeper investigation to reach the ultimate performances of MATISSE. The first one is the calibration of the effects of the chromatic OPD on the differential phase. The fundamental noise limits on the differential phase are well below $0.1^{\circ}$ and the current limit is of $1^{\circ}$ in $\mathrm{L}$ and $\mathrm{M}$ for local differential phase and up to $5^{\circ}$ near the band limits. A correct calibration of the differential phase in LR would open the way for chromatic imaging algorithms that use the differential phase and the coherent flux instead of the visibility and closure phase. This would extend the possibility to make $\mathrm{N}$ band images by 1 to 2 magnitudes. This new image reconstruction algorithm is therefore the second development axis. Finally, it appears that the main limitation of MATISSE with the current VLTI will be the sensitivity of the Adaptive Optics. MATISSE will be a major benefactor of the GRAVITY+ upgrade of the adaptive optics on UT as well as of the possibility to use off-axis guide stars for fringe tracking on UTs and ATs with the large isopistonic angle of the $\mathrm{N}$ band.

\section{REFERENCES}

[1] Xavier Haubois, et al, "VLTI status update: tapping into a powerful second-generation instrumentation," Proc. SPIE 11446, (2020).

[2] Frank Eisenhauer, “A new era of interferometry with GRAVITY,” Proc. SPIE 11443, (2020).

[3] Gravity collaboration et al, "First light for GRAVITY: Phase referencing optical interferometry for the Very Large Telescope Interferometer," Astronomy \& Astrophysics 602, (2017).

[4] Bruno Lopez, et al, "MATISSE: perspective of imaging in the mid-infrared at the VLTI," Proc SPIE 7013, (2008).

[5] Bruno Lopez, et al, "MATISSE, the VLTI mid-infrared imaging spectro-interferometer," submitted to Astronomy \& Astrophysics, (2021).

[6] Florian Kirchschlager, et al, "First L band detection of hot exozodiacal dust with VLTI/MATISSE," Monthly Notices of the Royal Astronomical Society: Letters, Volume 499, Issue 1, pp. L47-L52 (2020).

[7] Jozsef Varga, et al, "The asymmetric inner disk of the Herbig Ae star HD 163296 in the eyes of VLTI/MATISSE: evidence for a vortex?," to appear in Astronomy \& Astrophysics, (2021).

[8] Florentin Millour, et al, "Science with MATISSE," Proc. SPIE 11446, (2020).

[9] Romain G. Petrov, et al, "AMBER, the near-infrared spectro-interferometric three-telescope VLTI instrument," Astronomy and Astrophysics 464(1), 1-12 (2007). 
[10] Florentin Millour, et al, "AMBER closure and differential phases: accuracy and calibration with a beam commutation," Proc SPIE 7013, (2008).

[11] Alexis Matter, et al, "Parasitic interference in classical and nulling stellar interferometry," Proc SPIE 8445, (2012).

[12] Pierre Cruzalèbes, et al, "A catalogue of stellar diameters and fluxes for mid-infrared interferometry," Monthly Notices of the Royal Astronomical Society 490(3), 3158-3176 (2019).

[13] Philippe Berio, et al, "A posteriori co-phasing of $N$ band MATISSE fringes with the fringe tracker GRA4MAT," in preparation for Astronomy \& Astrophysics, (2021).

[14] Chris Koresko, et al, "Water vapor measurement and compensation in the near- and mid-infrared with the Keck Interferometer Nuller,” Proc SPIE 6268, (2006).

[15] Karl-Heinz Hofmann, et al, "Image reconstruction method IRBis for optical/infrared long-baseline interferometry," Proc SPIE 9907, (2016).

[16] Eric Thiébaud, "MIRA: an effective imaging algorithm for optical interferometry," Proc SPIE 7013, (2008).

[17] Jacques Kluska, et al, "First images from the PIONIER/VLTI optical interferometry imaging survey of Herbig Ae/Be stars," Proc IAU Symposium 299, (2014).

[18] Martin Vannier, et al, "Colour-differential interferometry for the observation of extrasolar planets,", Monthly Notices of the Royal Astronomical Society, Volume 367, Issue 2, pp. 825-837 (2006). 